\title{
Synthesis and styrene copolymerization of novel dimethyl and dimethoxy ring-substituted isobutyl phenylcyanoacrylates
}

Alessandra Cimino, Ehxciquiel M. Camacho, Samantha M. Evans, Daniela Garza, Dylan A.

Gregory, Julia C. Petrescu, Margaux J.E. Rocha, Brian J. Scannell, Katelyn M. Schreck, Mikal Zuljevic, Sara M. Rocus, William S. Schjerven and Gregory B. Kharas

DePaul University, Chemistry and Biochemistry Department, 1110 West Belden Avenue, Chicago, IL 60614-3214

Contact E-mail: gkharas@depaul.edu

\begin{abstract}
Novel dimethyl and dimethoxy ring-disubstituted isobutyl phenylcyanoacrylates, $\mathrm{RPhCH}=\mathrm{C}(\mathrm{CN}) \mathrm{CO}_{2} \mathrm{CH}_{2} \mathrm{CH}\left(\mathrm{CH}_{3}\right)_{2}$, where $\mathrm{R}$ is 2,3-dimethyl, 2,4-dimethyl, 3,5-dimethyl, 2,3-dimethoxy, 2,4-dimethoxy, 2,5-dimethoxy, 2,6-dimethoxy, 3,4-dimethoxy, 3,5dimethoxy were synthesized by the piperidine catalyzed Knoevenagel condensation of ringdisubstituted benzaldehydes and isobutyl cyanoacetate and characterized by $\mathrm{CHN}$ analysis, IR, ${ }^{1} \mathrm{H}$ and ${ }^{13} \mathrm{C} \mathrm{NMR}$. The acrylates were copolymerized with styrene in solution with
\end{abstract}


radical initiation $(\mathrm{ABCN})$ at $70^{\circ} \mathrm{C}$. The compositions of the copolymers were calculated from nitrogen analysis and the structures were analyzed by FTIR, ${ }^{1} \mathrm{H}$ and ${ }^{13} \mathrm{C}$ NMR.

\section{Introduction}

Dimethyl ring-substituted phenylcyanoacrylates (PCA) are reported in a number of applications [1-12]. Thus, 2,3-dimethyl-phenyl ethyl PCA was prepared inside microflow reactor using polymer networks carrying tertiary amine as a catalyst [1], whereas 3,4dimethyl-phenyl PCA was prepared with amine-functionalized polyacrylonitrile fiber [2]. This PCA was also reported in synthesis of highly substituted isoquinoline derivatives [3], in annulation/oxidation of cyclic amidines with activated olefins [4], and in asymmetric cyclopropanation of 2-cyano-3-arylacrylates with 2-bromomalonates [5]. 2,3Dimethoxy ring-disubstituted ethyl PCA used in synthesis of pyranoquinoline [6], of polysubstituted cyclopropanes [7], of some derivatives of o-vanillin [8], as well as in microwave enhanced Knoevenagel condensation of ethyl cyanoacetate with aldehydes [9]. 3,4-Dimethoxy-phenyl ring-disubstituted isobutyl PCA was reported in esterification and amide formation via acid-catalyzed dehydration and Ritter reactions [10]. 3,5-

Dimethoxy-phenyl methyl PCA was used in synthesis of pyrido[2,1-c]-1,2,4-triazine, 1,2, 4-triazolo[4,3-a]pyridine and 2-(substituted-pyrazolyl)nicotinonitrile [11] and in synthesis of dihydrouracils spiro-fused to pyrrolidines [12]. 
We have reported synthesis and styrene copolymerization a number of dimethyl and dimethoxy ring-disubstituted PCAs, such esters as methyl [13,14], ethyl [15-17], propyl [18, 19], isopropyl [20-22], and butyl [23, 24], 2-methoxyethyl [25], and octyl [26].

In this work we have prepared dimethyl and dimethoxy ring-disubstituted isobutyl PCA, $\mathrm{RPhCH}=\mathrm{C}(\mathrm{CN}) \mathrm{CO}_{2} \mathrm{CH}_{2} \mathrm{CH}\left(\mathrm{CH}_{3}\right)_{2}$, where $\mathrm{R}$ is 2,3-dimethyl, 2,4-dimethyl, 3,5-dimethyl, 2,3-dimethoxy, 2,4-dimethoxy, 2,5-dimethoxy, 2,6-dimethoxy, 3,4-dimethoxy, 3,5dimethoxy, and radically copolymerized them with styrene. To the best of our knowledge, there have been no reports (except 3,4-dimethoxy [10]) on either synthesis of these compounds, nor their copolymerization with styrene [27].

\section{Experimental}

2,3-Dimethyl, 2,4-dimethyl, 3,5-dimethyl, 2,3-dimethoxy, 2,4-dimethoxy, 2,5-dimethoxy, 2,6-dimethoxy, 3,4-dimethoxy, 3,5-dimethoxybenzaldehydes, isobutyl cyanoacetate, piperidine, styrene, 1,1'-azobis(cyclohexanecarbonitrile) (ABCN), and toluene supplied from Sigma-Aldrich Co., were used as received. Instrumentation is described in [28].

\section{Results and discussion}

\subsection{Synthesis and characterization of isobutyl phenylcyanoacrylates}

All isobutyl phenylcyanoacrylates (IPCA) compounds were synthesized by Knoevenagel condensation [29] of appropriate benzaldehydes with isobutyl cyanoacetate, catalyzed by base, piperidine (Scheme 1). 


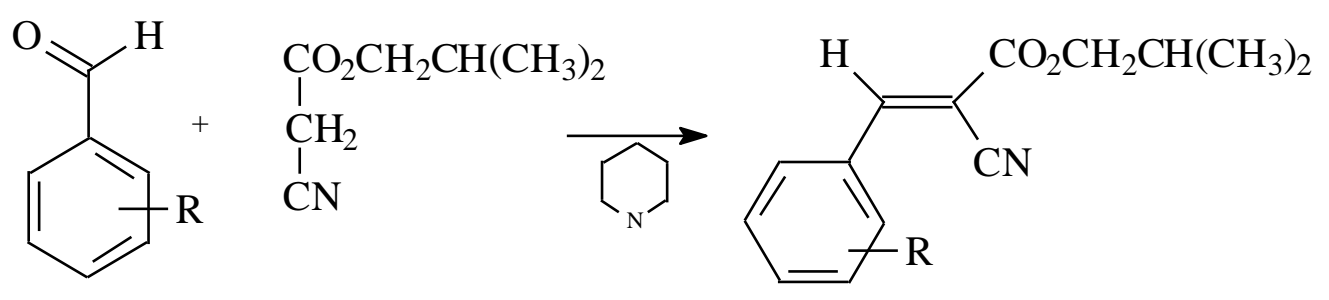

Scheme 1. Synthesis of isobutyl R-phenylcyanoacrylates, where R is 2,3-dimethyl, 2,4dimethyl, 3,5-dimethyl, 2,3-dimethoxy, 2,4-dimethoxy, 2,5-dimethoxy, 2,6-dimethoxy, 3,4dimethoxy, 3,5-dimethoxy.

The preparation procedure was essentially the same for all the monomers. In a typical synthesis, equimolar amounts of isobutyl cyanoacetate and an appropriate benzaldehyde were mixed in equimolar ratio in a $20 \mathrm{~mL}$ vial. A few drops of piperidine were added with stirring. The product of the reaction was isolated by filtration and purified by crystallization from 2-propanol. The condensation reaction proceeded smoothly, yielding products, which were purified by conventional techniques. Melting points of the compounds in crystalline state were measured by DSC. The compounds were characterized by $\mathrm{IR},{ }^{1} \mathrm{H}$ and ${ }^{13} \mathrm{C} \mathrm{NMR}$ spectroscopies. No stereochemical analysis of the novel ring-substituted IPCA was performed since no stereoisomers $(E$ or/and $Z$ ) of known configuration were available.

\subsubsection{Isobutyl 2,3-dimethylphenylcyanoacrylate}

Yield: $83.6 \%$; mp $52.9^{\circ} \mathrm{C} ;{ }^{1} \mathrm{H}$ NMR: $\delta 8.6(\mathrm{~s}, 1 \mathrm{H}, \mathrm{CH}=), 8.0-7.0(\mathrm{~m}, 4 \mathrm{H}, \mathrm{Ph}), 4.1(\mathrm{~d}, 2 \mathrm{H}$, $\left.\mathrm{CH}_{2}\right), 2.3(\mathrm{~m}, 1 \mathrm{H}, \mathrm{CH}), 2.1\left(\mathrm{~s}, 6 \mathrm{H}, \mathrm{CH}_{3}\right), 1.0\left(\mathrm{~d}, 6 \mathrm{H}, \mathrm{CH}_{3}\right) ;{ }^{13} \mathrm{C} \mathrm{NMR}: \delta 162(\mathrm{C}=\mathrm{O}), 155$ $(\mathrm{HC}=), 137,134,130,122(\mathrm{Ph}), 115(\mathrm{CN}), 107(\mathrm{C}=), 73\left(\mathrm{CH}_{2}\right), 63\left(\mathrm{OCH}_{3}\right), 28(\mathrm{CH}), 20$ $\left(\mathrm{CH}_{3}\right)$ 16,20 (PhCH 3$)$; IR: (cm-1) 3024-2822 (m, C-H), $2225(\mathrm{~m}, \mathrm{CN}), 1728$ (s, C=O), 
1587 (s, C=C), 1286 (s, C-O-CH $\left.{ }_{3}\right), 793,760,710$ (s, C-H out of plane). Anal. calcd. for

$\mathrm{C}_{16} \mathrm{H}_{19} \mathrm{NO}_{2}: \mathrm{C}, 74.68 ; \mathrm{H}, 7.44 ; \mathrm{N}, 5.44$; Found: C, 72.76; H, 7.21; N, 5.48.

\subsubsection{Isobutyl 2,4-dimethylphenylcyanoacrylate}

Yield $82 \%$; mp $49.8^{\circ} \mathrm{C} ;{ }^{1} \mathrm{H}$ NMR $\delta 8.5(\mathrm{~s}, 1 \mathrm{H}, \mathrm{CH}=), 8.2-6.8(\mathrm{~m}, 4 \mathrm{H}, \mathrm{Ph}), 4.1(\mathrm{~d}, 2 \mathrm{H}$,

$\left.\mathrm{CH}_{2}\right), 2.4(\mathrm{~m}, 1 \mathrm{H}, \mathrm{CH}), 2.3\left(\mathrm{~s}, 6 \mathrm{H}, \mathrm{CH}_{3}\right), 1.0\left(\mathrm{~d}, 6 \mathrm{H}, \mathrm{CH}_{3}\right) ;{ }^{13} \mathrm{C}$ NMR $\delta 163(\mathrm{C}=\mathrm{O}), 152$

$(\mathrm{HC}=), 143,138,133,128,124(\mathrm{Ph}), 117(\mathrm{CN}), 103(\mathrm{C}=), 72\left(\mathrm{CH}_{2}\right), 56\left(\mathrm{OCH}_{3}\right), 31$

(CH), 19-15 ( $\left.\mathrm{CH}_{3}\right)$; IR $\left(\mathrm{cm}^{-1}\right)$ : 3234-2887, $2224(\mathrm{~m}, \mathrm{CN}), 1726$ (s, C=O), $1595(\mathrm{C}=\mathrm{C})$,

1288 (s, C-O- $\mathrm{CH}_{3}$ ), 824, 784 (s, C-H out of plane). Anal. Calcd. for $\mathrm{C}_{16} \mathrm{H}_{19} \mathrm{NO}_{2}$ : C,

74.68; H, 7.44; N, 5.44; Found: C, 71.57; H, 7.33; N, 5.80.

\subsubsection{Isobutyl 3,5-dimethylphenylcyanoacrylate}

Yield 78\%; mp $122.3^{\circ} \mathrm{C} ;{ }^{1} \mathrm{H}$ NMR $\delta 8.2(\mathrm{~s}, 1 \mathrm{H}, \mathrm{CH}=), 7.7-7.0(\mathrm{~m}, 4 \mathrm{H}, \mathrm{Ph}), 4.1(\mathrm{~d}, 2 \mathrm{H}$,

$\left.\mathrm{CH}_{2}\right), 2.3\left(\mathrm{~s}, 6 \mathrm{H}, \mathrm{CH}_{3} \mathrm{Ph}\right), 2.1(\mathrm{~m}, 1 \mathrm{H}, \mathrm{CH}), 1.0\left(\mathrm{~d}, 6 \mathrm{H}, \mathrm{CH}_{3}\right) ;{ }^{13} \mathrm{C} \mathrm{NMR} \delta 163(\mathrm{C}=\mathrm{O})$, $153(\mathrm{HC}=), 139,135,133,124,119(\mathrm{Ph}), 115(\mathrm{CN}), 102(\mathrm{C}=), 72\left(\mathrm{CH}_{2}\right), 28\left(\mathrm{CH}_{3}\right), 28$

(CH), $21\left(\mathrm{CH}_{3} \mathrm{Ph}\right), 19\left(\mathrm{CH}_{3}\right)$; IR ( $\left.\mathrm{cm}^{-1}\right)$ : 3045-2937 (m, C-H), 2216 (m, CN), 1713 (s, $\mathrm{C}=\mathrm{O}), 1595(\mathrm{C}=\mathrm{C}), 1229$ (s, C-O-CH3), 837 (s, C-H out of plane). Anal. Calcd. for $\mathrm{C}_{16} \mathrm{H}_{19} \mathrm{NO}_{2}: \mathrm{C}, 74.68 ; \mathrm{H}, 7.44 ; \mathrm{N}, 5.44$; Found: C, 74.23; H, 7.28; N, 5.89.

\subsubsection{Isobutyl 2,3-dimethoxyphenylcyanoacrylate}

Yield 88\%; mp 78.3 ${ }^{\circ} \mathrm{C} ;{ }^{1} \mathrm{H}$ NMR $\delta 8.7(\mathrm{~s}, 1 \mathrm{H}, \mathrm{CH}=), 8.0-6.8(\mathrm{~m}, 4 \mathrm{H}, \mathrm{Ph}), 4.1(\mathrm{~m}, 2 \mathrm{H}$, $\left.\mathrm{CH}_{2}\right), 3.8\left(\mathrm{~s}, 6 \mathrm{H}, \mathrm{OCH}_{3}\right), 2.1(\mathrm{~m}, 1 \mathrm{H}, \mathrm{CH}), 1.0\left(\mathrm{~d}, 6 \mathrm{H},\left(\mathrm{CH}_{3}\right)_{2}\right) ;{ }^{13} \mathrm{C} \mathrm{NMR} \delta 163(\mathrm{C}=\mathrm{O})$, $152(\mathrm{HC}=), 149,129,123,122(\mathrm{Ph}), 116(\mathrm{CN}), 103(\mathrm{C}=), 72\left(\mathrm{CH}_{2}\right), 61,55\left(\mathrm{CH}_{3} \mathrm{O}\right), 27$ (CH), $18\left(\mathrm{CH}_{3}\right)_{2}$; IR ( $\left.\mathrm{cm}^{-1}\right)$ : 3120-2918 (m, C-H), 2222 (m, CN), 1728 (s, C=O), 1605 (s, 
$\mathrm{C}=\mathrm{C}$ ), 1242 (s, $\left.\mathrm{C}-\mathrm{O}-\mathrm{CH}_{3}\right), 764$ (s, $\mathrm{C}-\mathrm{H}$ out of plane). Anal. Calcd. for $\mathrm{C}_{16} \mathrm{H}_{19} \mathrm{NO}_{4}: \mathrm{C}$, 66.42; H, 6.62; N, 4.84; Found: C, 65.07; H, 6.53; N, 4.88.

\subsubsection{Isobutyl 2,4-dimethoxyphenylcyanoacrylate}

Yield 91\%; mp 82.7 $\mathrm{C} ;{ }^{1} \mathrm{H}$ NMR $\delta 8.6(\mathrm{~s}, 1 \mathrm{H}, \mathrm{CH}=), 8.5-8.3(\mathrm{~m}, 4 \mathrm{H}, \mathrm{Ph}), 4.1(\mathrm{~m}, 2 \mathrm{H}$, $\left.\mathrm{CH}_{2}\right), 3.9\left(\mathrm{~s}, 6 \mathrm{H}, \mathrm{OCH}_{3}\right), 2.1(\mathrm{~m}, 1 \mathrm{H}, \mathrm{CH}), 1.0\left(\mathrm{~d}, 6 \mathrm{H},\left(\mathrm{CH}_{3}\right)_{2}\right) ;{ }^{13} \mathrm{C} \mathrm{NMR} \delta 166(\mathrm{C}=\mathrm{O})$, $162(\mathrm{HC}=), 161,148,131(\mathrm{Ph}), 117(\mathrm{CN}), 106(\mathrm{C}=), 72\left(\mathrm{CH}_{2}\right), 56\left(\mathrm{CH}_{3} \mathrm{O}\right), 28(\mathrm{CH}), 20$ $\left(\mathrm{CH}_{3}\right)_{2}$; IR ( $\left.\mathrm{cm}^{-1}\right)$ : 2910(m, C-H), 2223 (m, CN), 1722 (s, C=O), 1607 (s, C=C), 1261 (s, C-O- $\mathrm{CH}_{3}$ ), 781, 762 (s, C-H out of plane). Anal. Calcd. for $\mathrm{C}_{16} \mathrm{H}_{19} \mathrm{NO}_{4}$ : C, 66.42; H, 6.62; N, 4.84; Found: C, 66.07; H, 6.54; N, 4.98.

\subsubsection{Isobutyl 2,5-dimethoxyphenylcyanoacrylate}

Yield 76\%; mp 78.1 ${ }^{\circ} \mathrm{C} ;{ }^{1} \mathrm{H}$ NMR $\delta 8.7(\mathrm{~s}, 1 \mathrm{H}, \mathrm{CH}=), 7.8-7.2(\mathrm{~m}, 4 \mathrm{H}, \mathrm{Ph}), 4.4(\mathrm{~m}, 2 \mathrm{H}$,

$\left.\mathrm{CH}_{2}\right), 3.8\left(\mathrm{~s}, 6 \mathrm{H}, \mathrm{OCH}_{3}\right), 2.4(\mathrm{~m}, 1 \mathrm{H}, \mathrm{CH}), 1.0\left(\mathrm{~d}, 6 \mathrm{H},\left(\mathrm{CH}_{3}\right)_{2}\right) ;{ }^{13} \mathrm{C} \mathrm{NMR} \delta 163(\mathrm{C}=\mathrm{O})$, $152(\mathrm{HC}=), 152,148,131,127(\mathrm{Ph}), 115(\mathrm{CN}), 102(\mathrm{C}=), 71\left(\mathrm{CH}_{2}\right), 56\left(\mathrm{CH}_{3} \mathrm{O}\right), 28(\mathrm{CH})$, $19\left(\mathrm{CH}_{3}\right)_{2}$; IR ( $\left.\mathrm{cm}^{-1}\right): 2918(\mathrm{~m}, \mathrm{C}-\mathrm{H}), 2223(\mathrm{~m}, \mathrm{CN}), 1729$ (s, C=O), 1647 (s, C=C), 1263 (s, C-O- $\mathrm{CH}_{3}$ ), 786 (s, C-H out of plane). Anal. Calcd. for $\mathrm{C}_{16} \mathrm{H}_{19} \mathrm{NO}_{4}$ : C, 66.42; H, 6.62; N, 4.84; Found: C, 65.73; H, 6.53; N, 4.99.

\subsubsection{Isobutyl 2,6-dimethoxyphenylcyanoacrylate}

Yield 76\%; mp 85.4 ${ }^{\circ} \mathrm{C} ;{ }^{1} \mathrm{H}$ NMR $\delta 8.4(\mathrm{~s}, 1 \mathrm{H}, \mathrm{CH}=), 7.7-6.4(\mathrm{~m}, 4 \mathrm{H}, \mathrm{Ph}), 4.1(\mathrm{~m}, 2 \mathrm{H}$, $\left.\mathrm{CH}_{2}\right), 3.9\left(\mathrm{~s}, 6 \mathrm{H}, \mathrm{OCH}_{3}\right), 2.1(\mathrm{~m}, 1 \mathrm{H}, \mathrm{CH}), 1.0\left(\mathrm{~d}, 6 \mathrm{H},\left(\mathrm{CH}_{3}\right)_{2} ;{ }^{13} \mathrm{C} \mathrm{NMR} \delta 163(\mathrm{C}=\mathrm{O})\right.$, $159(\mathrm{HC}=)$, 148, 133, 110, $107(\mathrm{Ph}), 115(\mathrm{CN}), 104(\mathrm{C}=), 72\left(\mathrm{CH}_{2}\right), 55\left(\mathrm{CH}_{3} \mathrm{O}\right), 28(\mathrm{CH})$, $19\left(\mathrm{CH}_{3}\right)_{2}$; IR ( $\left.\mathrm{cm}^{-1}\right): 3120-2818(\mathrm{~m}, \mathrm{C}-\mathrm{H}), 2225(\mathrm{~m}, \mathrm{CN}), 1722$ (s, C=O), 1607 (s, C=C), 
1288 (s, C-O- $\mathrm{CH}_{3}$ ), 781, 762 (s, C-H out of plane). Anal. Calcd. for $\mathrm{C}_{16} \mathrm{H}_{19} \mathrm{NO}_{4}$ : C, 66.42; H, 6.62; N, 4.84; Found: C, 64.03; H, 6.43; N, 4.76.

\subsubsection{Isobutyl 2,5-dimethoxyphenylcyanoacrylate}

Yield 76\%; mp 86.9 ${ }^{\circ}$; ${ }^{1} \mathrm{H}$ NMR $\delta 8.2(\mathrm{~s}, 1 \mathrm{H}, \mathrm{CH}=), 8.0-6.9(\mathrm{~m}, 4 \mathrm{H}, \mathrm{Ph}), 4.5(\mathrm{~m}, 2 \mathrm{H}$, $\left.\mathrm{CH}_{2}\right), 4.0\left(\mathrm{~s}, 6 \mathrm{H}, \mathrm{OCH}_{3}\right), 2.1(\mathrm{~m}, 1 \mathrm{H}, \mathrm{CH}), 1.0\left(\mathrm{~d}, 6 \mathrm{H},\left(\mathrm{CH}_{3}\right)_{2} ;{ }^{13} \mathrm{C} \mathrm{NMR} \delta 162(\mathrm{C}=\mathrm{O})\right.$, $154(\mathrm{HC}=), 153,148,131,127,124,110(\mathrm{Ph}), 115(\mathrm{CN}), 99(\mathrm{C}=), 71\left(\mathrm{CH}_{2}\right), 55\left(\mathrm{CH}_{3} \mathrm{O}\right)$, $27(\mathrm{CH}), 19\left(\mathrm{CH}_{3}\right)_{2} ; \mathrm{IR}\left(\mathrm{cm}^{-1}\right): 3102-2818(\mathrm{~m}, \mathrm{C}-\mathrm{H}), 2217(\mathrm{~m}, \mathrm{CN}), 1722(\mathrm{~s}, \mathrm{C}=\mathrm{O})$, 1589 (s, C=C), 1263 (s, C-O-CH 3 ), 786 (s, C-H out of plane). Anal. Calcd. for $\mathrm{C}_{16} \mathrm{H}_{19} \mathrm{NO}_{4}: \mathrm{C}, 66.42 ; \mathrm{H}, 6.62 ; \mathrm{N}, 4.84$; Found: C, 64.71; H, 6.53; N, 4.45.

\subsubsection{Isobutyl 3,5-dimethoxyphenylcyanoacrylate}

Yield $84 \%$; mp $104.1^{\circ} \mathrm{C} ;{ }^{1} \mathrm{H}$ NMR $\delta 8.2(\mathrm{~s}, 1 \mathrm{H}, \mathrm{CH}=), 7.4-6.5(\mathrm{~m}, 4 \mathrm{H}, \mathrm{Ph}), 4.1(\mathrm{~m}, 2 \mathrm{H}$, $\left.\mathrm{CH}_{2}\right), 4.0\left(\mathrm{~s}, 6 \mathrm{H}, \mathrm{OCH}_{3}\right), 2.1(\mathrm{~m}, 1 \mathrm{H}, \mathrm{CH}), 1.0\left(\mathrm{~d}, 6 \mathrm{H},\left(\mathrm{CH}_{3}\right)_{2}\right) ;{ }^{13} \mathrm{C} \mathrm{NMR} \delta 162(\mathrm{C}=\mathrm{O})$, $155(\mathrm{HC}=)$, 154, 133, 108, $106(\mathrm{Ph}), 115(\mathrm{CN}), 103(\mathrm{C}=), 73\left(\mathrm{CH}_{2}\right), 56\left(\mathrm{CH}_{3} \mathrm{O}\right), 28(\mathrm{CH})$, $19\left(\mathrm{CH}_{3}\right)_{2}$; IR (cm $\left.{ }^{-1}\right): 3215$ - $2812(\mathrm{~m}, \mathrm{C}-\mathrm{H}), 2221(\mathrm{~m}, \mathrm{CN}), 1717$ (s, C=O), 1609 (s, $\mathrm{C}=\mathrm{C}), 1240$ (s, C-O-CH$\left.{ }_{3}\right), 785,761$ (s, C-H out of plane). Anal. Calcd. for $\mathrm{C}_{16} \mathrm{H}_{19} \mathrm{NO}_{4}$ : C, 66.42; H, 6.62; N, 4.84; Found: C, 65.18; H, 6.46; N, 4.80.

\subsection{Synthesis and characterization of styrene - IPCA copolymers}

Copolymers of the styrene (ST) and the IPCA compounds, P(ST-co-IPCA) were prepared in $25-\mathrm{mL}$ glass screw cap vials at ST/IPCA $=3(\mathrm{~mol})$ the monomer feed using $0.12 \mathrm{~mol} / \mathrm{L}$ of $\mathrm{ABCN}$ at an overall monomer concentration $2.44 \mathrm{~mol} / \mathrm{L}$ in $10 \mathrm{~mL}$ of 
toluene. The copolymerization was conducted at $70^{\circ} \mathrm{C}$. After a predetermined time, the mixture was cooled to room temperature, and precipitated dropwise in methanol. The composition of the copolymers was determined based on the nitrogen content. The novel synthesized IPCA compounds copolymerized readily with ST under free-radical conditions (Scheme 2) forming white flaky precipitates when their solutions were poured into methanol. The conversion of the copolymers was kept between 10 and $20 \%$ to minimize compositional drift (Table 1). Nitrogen elemental analysis showed that between 18.1 and $30.1 \mathrm{~mol} \%$ of IPCA is present in the copolymers, which is indicative of relatively high reactivity of the IPCA monomers towards ST radical which is typical of dimethyl and dimethoxy ring-disubstituted different esters PCA [13-26]. Since IPCA monomers do not homopolymerize, the most likely structure of the copolymers would be isolated IPCA monomer $(\mathrm{y}=1)$ units alternating with short ST sequences $(\mathrm{x}>1)$ (Scheme 2).

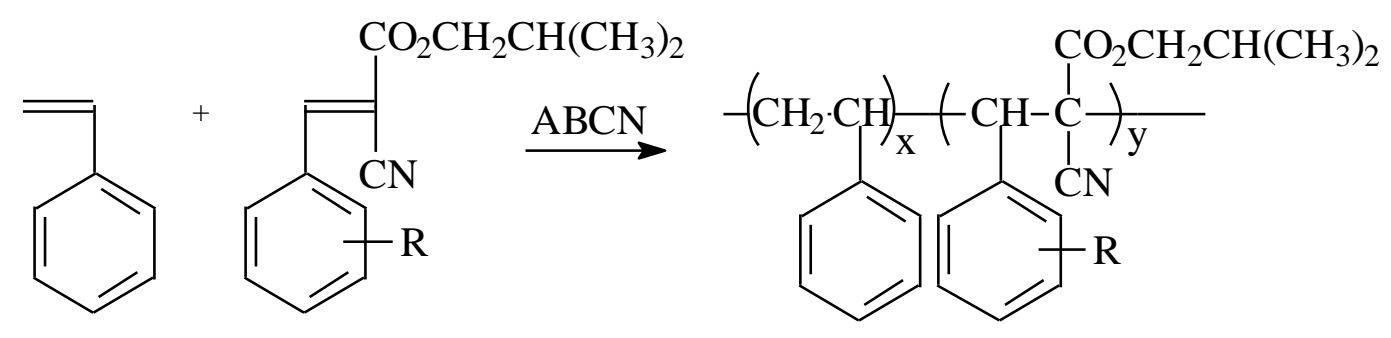

Scheme 2. Copolymerization of ST and the ring-disubstituted isobutyl

phenylcyanoacrylates, $\mathrm{RPhCH}=\mathrm{C}(\mathrm{CN}) \mathrm{CO}_{2} \mathrm{CH}_{2} \mathrm{CH}\left(\mathrm{CH}_{3}\right)_{2}, \mathrm{R}=$ 2,3-dimethyl, 2,4-

dimethyl, 3,5-dimethyl, 2,3-dimethoxy, 2,4-dimethoxy, 2,5-dimethoxy, 2,6-dimethoxy, 3,4dimethoxy, 3,5-dimethoxy. 


\subsubsection{Styrene- Isobutyl 2,3-dimethylphenylcyanoacrylate copolymer}

Yield 11\%; ${ }^{1} \mathrm{H}-\mathrm{NMR} \delta 7.4-6.3(8 \mathrm{H}, \mathrm{Ph}), 5.2-5.0(1 \mathrm{H}, \mathrm{OCH}), 4.1-3.7(1 \mathrm{H}, \mathrm{CHPh}-$ ST), 1.6 - $1.4\left(2 \mathrm{H}, \mathrm{CH}_{2}\right), 2.7-2.4\left(6 \mathrm{H}, \mathrm{PhCH}_{3}\right), 2.7$ - 2.4 (1H, CH-ICPP), 1.5-1.0 (6H, $\left.\mathrm{CH}\left(\mathrm{CH}_{3}\right)_{2}\right) ;{ }^{13} \mathrm{C}-\mathrm{NMR} \delta 174-165(\mathrm{C}=\mathrm{O}), 152-100(\mathrm{Ph}), 118-114(\mathrm{CN}), 68-58(\mathrm{OCH})$, 51-41 (CH, $\left.\mathrm{CH}_{2}\right), 24-19\left(\mathrm{CH}\left(\mathrm{CH}_{3}\right)_{2}\right), 21-14\left(\mathrm{CH}_{3}\right)$; IR $\left(\mathrm{cm}^{-1}\right)$ : 3023-2822 (m, C-H), 2239 (m, CN), 1741 (s, C=O), 1249 (s, C-O-C), 778 (s, C-H out of plane). Nitrogen content $2.12 \%$.

\subsubsection{Styrene - Isobutyl 2,4-dimethylphenylcyanoacrylate copolymer} Yield 13\%; ${ }^{1} \mathrm{H}-\mathrm{NMR} \delta 7.5-6.6(8 \mathrm{H}, \mathrm{Ph}), 5.3-5.1(1 \mathrm{H}, \mathrm{OCH}), 4.0-3.6(1 \mathrm{H}, \mathrm{CHPh}-$ ST), 1.5 - $1.3\left(2 \mathrm{H}, \mathrm{CH}_{2}\right), 2.6$ - $2.3\left(6 \mathrm{H}, \mathrm{PhCH}_{3}\right), 2.6$ - $2.4(1 \mathrm{H}, \mathrm{CH}-\mathrm{ICPP}), 1.4$ - $0.9(6 \mathrm{H}$, $\left.\mathrm{CH}\left(\mathrm{CH}_{3}\right)_{2}\right) ;{ }^{13} \mathrm{C}-\mathrm{NMR} \delta$ 173-164 (C=O), $151-101(\mathrm{Ph}), 119$ - $112(\mathrm{CN}), 68$ - $57(\mathrm{OCH})$, $51-40\left(\mathrm{CH}, \mathrm{CH}_{2}\right), 25-20\left(\mathrm{CH}\left(\mathrm{CH}_{3}\right)_{2}\right), 21-14\left(\mathrm{CH}_{3}\right) ; \mathrm{IR}\left(\mathrm{cm}^{-1}\right): 3012-2825(\mathrm{~m}, \mathrm{C}-\mathrm{H})$, $2242(\mathrm{~m}, \mathrm{CN}), 1740$ (s, C=O), 1229 (s, C-O-C), 750 (s, C-H out of plane). Nitrogen content $1.92 \%$.

\subsubsection{Styrene - Isobutyl 3,5-dimethylphenylcyanoacrylate copolymer}

Yield 12\%; ${ }^{1} \mathrm{H}-\mathrm{NMR} \delta 7.6-6.5(8 \mathrm{H}, \mathrm{Ph}), 5.3-5.0(1 \mathrm{H}, \mathrm{OCH}), 4.0-3.7(1 \mathrm{H}, \mathrm{CHPh}-$ ST), 1.6 - $1.4\left(2 \mathrm{H}, \mathrm{CH}_{2}\right), 2.7-2.4\left(6 \mathrm{H}, \mathrm{PhCH}_{3}\right), 2.6$ - 2.5 (1H, CH-ICPP), 1.4-1.0 (6H, $\left.\mathrm{CH}\left(\mathrm{CH}_{3}\right)_{2}\right) ;{ }^{13} \mathrm{C}-\mathrm{NMR} \delta$ 171-165 $(\mathrm{C}=\mathrm{O}), 152-100(\mathrm{Ph}), 118-116(\mathrm{CN}), 68-58(\mathrm{OCH})$, 51-41 (CH, $\left.\mathrm{CH}_{2}\right), 24-19\left(\mathrm{CH}\left(\mathrm{CH}_{3}\right)_{2}\right), 22$ - $14\left(\mathrm{CH}_{3}\right) ; \mathrm{IR}\left(\mathrm{cm}^{-1}\right): 3128-2832(\mathrm{~m}, \mathrm{C}-\mathrm{H})$, 2243 (m, CN), 1742 (s, C=O), 1268 (s, C-O-C), 853, 760, 738 (s, C-H out of plane). Nitrogen content $2.63 \%$. 


\subsubsection{Styrene - Isobutyl 2,3-dimethoxyphenylcyanoacrylate copolymer}

Yield 12\%; ${ }^{1} \mathrm{H}-\mathrm{NMR} \delta 7.5-6.6(8 \mathrm{H}, \mathrm{Ph}), 5.3-5.1(1 \mathrm{H}, \mathrm{OCH}), 4.1-3.7(1 \mathrm{H}, \mathrm{CHPh}-$

ST), 3.8-3.5 (6H, $\left.\mathrm{PhOCH}_{3}\right), 2.7$ - $2.5(1 \mathrm{H}, \mathrm{CH}-\mathrm{ICPP}), 1.5$ - 1.3 (2H, $\left.\mathrm{CH}_{2}\right), 1.6-1.1(6 \mathrm{H}$, $\left.\mathrm{CH}\left(\mathrm{CH}_{3}\right)_{2}\right) ;{ }^{13} \mathrm{C}-\mathrm{NMR} \delta$ 176-166 (C=O), $151-99(\mathrm{Ph}), 117$ - $115(\mathrm{CN}), 69-58(\mathrm{OCH})$, 61-54 ( $\left.\mathrm{CH}_{3} \mathrm{OPh}\right)$, 52-42 (CH, $\left.\mathrm{CH}_{2}\right), 23-18\left(\mathrm{CH}_{(}\left(\mathrm{CH}_{3}\right)_{2}\right)$; IR $\left(\mathrm{cm}^{-1}\right): 3145-2812(\mathrm{~m}, \mathrm{C}-\mathrm{H})$, 2239 (m, CN), 1742 (s, C=O), 1266 (s, C-O-C), 754, 698 (s, C-H out of plane). Nitrogen content $2.3 \%$.

\subsubsection{Styrene - Isobutyl 2,4-dimethoxyphenylcyanoacrylate copolymer}

Yield 10\%; ${ }^{1} \mathrm{H}-\mathrm{NMR} \delta 7.6-6.6(8 \mathrm{H}, \mathrm{Ph}), 5.3-5.1(1 \mathrm{H}, \mathrm{OCH}), 4.0-3.6(1 \mathrm{H}, \mathrm{CHPh}-$ ST), 3.7-3.5 (6H, $\left.\mathrm{PhOCH}_{3}\right), 2.7$ - $2.5(1 \mathrm{H}, \mathrm{CH}-\mathrm{ICPP}), 1.5$ - $1.3\left(2 \mathrm{H}, \mathrm{CH}_{2}\right), 1.4$ - $0.9(6 \mathrm{H}$, $\left.\mathrm{CH}\left(\mathrm{CH}_{3}\right)_{2}\right) ;{ }^{13} \mathrm{C}-\mathrm{NMR} \delta$ 172-165 (C=O), $154-101(\mathrm{Ph}), 118-114(\mathrm{CN}), 68-57(\mathrm{OCH})$, 61-54 ( $\left.\mathrm{CH}_{3} \mathrm{OPh}\right), 51-42\left(\mathrm{CH}, \mathrm{CH}_{2}\right), 25-21\left(\mathrm{CH}\left(\mathrm{CH}_{3}\right)_{2}\right) ; \mathrm{IR}\left(\mathrm{cm}^{-1}\right): 3068-2832(\mathrm{~m}, \mathrm{C}-$ H), 2239 (m, CN), 1742 (s, C=O), 1245 (s, C-O-C), 767 (s, C-H out of plane). Nitrogen content $1.87 \%$.

\subsubsection{Styrene - Isobutyl 2,5-dimethoxyphenylcyanoacrylate copolymer} Yield 12\%; ${ }^{1} \mathrm{H}-\mathrm{NMR} \delta 7.5-6.5(8 \mathrm{H}, \mathrm{Ph}), 5.4-5.1(1 \mathrm{H}, \mathrm{OCH}), 4.1-3.6(1 \mathrm{H}, \mathrm{CHPh}-$ ST), 3.7-3.5 (6H, $\left.\mathrm{PhOCH}_{3}\right), 1.6$ - $1.3\left(2 \mathrm{H}, \mathrm{CH}_{2}\right), 2.5$ - $2.3\left(6 \mathrm{H}, \mathrm{PhCH}_{3}\right), 2.6$ - $2.3(1 \mathrm{H}$, CH-ICPP), 1.3 - $0.9\left(6 \mathrm{H}, \mathrm{CH}\left(\mathrm{CH}_{3}\right)_{2}\right) ;{ }^{13} \mathrm{C}-\mathrm{NMR} \delta$ 173-163 (C=O), $153-101(\mathrm{Ph}), 118$ -

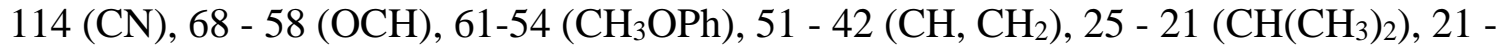
$14\left(\mathrm{CH}_{3}\right)$; FTIR $\left(\mathrm{cm}^{-1}\right): 3147-2835(\mathrm{~m}, \mathrm{C}-\mathrm{H}), 2239(\mathrm{~m}, \mathrm{CN}), 1740(\mathrm{~s}, \mathrm{C}=\mathrm{O}), 1223(\mathrm{~s}, \mathrm{C}-$ O-C), 767 (s, C-H out of plane). Nitrogen content $2.37 \%$. 


\subsubsection{Styrene - Isobutyl 2,6-dimethoxyphenylcyanoacrylate copolymer}

Yield 12\%; ${ }^{1} \mathrm{H}-\mathrm{NMR} \delta 7.5-6.6(8 \mathrm{H}, \mathrm{Ph}), 5.3-5.1(1 \mathrm{H}, \mathrm{OCH}), 4.1-3.7(1 \mathrm{H}, \mathrm{CHPh}-$

ST), 3.7-3.5 (6H, $\left.\mathrm{PhOCH}_{3}\right), 1.5-1.3\left(2 \mathrm{H}, \mathrm{CH}_{2}\right), 2.8-2.5\left(6 \mathrm{H}, \mathrm{PhCH}_{3}\right), 2.7-2.5(1 \mathrm{H}, \mathrm{CH}-$

ICPP), 1.6-1.1 (6H, $\left.\mathrm{CH}\left(\mathrm{CH}_{3}\right)_{2}\right) ;{ }^{13} \mathrm{C}-\mathrm{NMR} \delta$ 176-166 (C=O), 151 - $99(\mathrm{Ph}), 117$ - 115

(CN), 69-58 (OCH), 61-54 ( $\left.\mathrm{CH}_{3} \mathrm{OPh}\right)$, 52-42 (CH, $\left.\mathrm{CH}_{2}\right)$, 23-18 $\left(\mathrm{CH}\left(\mathrm{CH}_{3}\right)_{2}\right), 21$ - 14

$\left(\mathrm{CH}_{3}\right)$; FTIR $\left(\mathrm{cm}^{-1}\right)$ : 3145-2812 (m, C-H), $2239(\mathrm{~m}, \mathrm{CN}), 1742$ (s, C=O), 1266 (s, C-O-

C), 754, 698 (s, C-H out of plane). Nitrogen content 1.87\%.

\subsubsection{Styrene - Isobutyl 3,4-dimethoxyphenylcyanoacrylate copolymer}

Yield 12\%; ${ }^{1} \mathrm{H}-\mathrm{NMR} \delta 7.5-6.6(8 \mathrm{H}, \mathrm{Ph}), 5.3-5.1(1 \mathrm{H}, \mathrm{OCH}), 4.1-3.7(1 \mathrm{H}, \mathrm{CHPh}-$

ST), 3.7-3.5 (6H, $\left.\mathrm{PhOCH}_{3}\right), 1.5-1.3\left(2 \mathrm{H}, \mathrm{CH}_{2}\right), 2.8-2.5\left(6 \mathrm{H}, \mathrm{PhCH}_{3}\right), 2.7-2.5(1 \mathrm{H}, \mathrm{CH}-$

ICPP), 1.6-1.1 (6H, $\left.\mathrm{CH}\left(\mathrm{CH}_{3}\right)_{2}\right) ;{ }^{13} \mathrm{C}-\mathrm{NMR} \delta$ 176-166 (C=O), 151 - $99(\mathrm{Ph}), 117$ - 115

(CN), 69-58 (OCH), 61-54 ( $\left.\mathrm{CH}_{3} \mathrm{OPh}\right)$, 52-42 (CH, $\left.\mathrm{CH}_{2}\right)$, ,23-18 $\left(\mathrm{CH}\left(\mathrm{CH}_{3}\right)_{2}\right), 21$ - 14

$\left(\mathrm{CH}_{3}\right)$; FTIR $\left(\mathrm{cm}^{-1}\right)$ : 3141-2800 (m, C-H), $2243(\mathrm{~m}, \mathrm{CN}), 1741$ (s, C=O), 1263 (s, C-O-

C), 761, 737, 699 (s, C-H out of plane). Nitrogen content $2.15 \%$.

\subsubsection{Styrene - Isobutyl 3,5-dimethoxyphenylcyanoacrylate copolymer}

Yield 11\%; ${ }^{1} \mathrm{H}-\mathrm{NMR} \delta 7.6-6.3(8 \mathrm{H}, \mathrm{Ph}), 5.4-5.2(1 \mathrm{H}, \mathrm{OCH}), 4.2-3.5(1 \mathrm{H}, \mathrm{CHPh}-$

ST), 3.9-3.5 (6H, $\left.\mathrm{CH}_{3} \mathrm{O}\right), 3.7-3.5\left(6 \mathrm{H}, \mathrm{PhOCH}_{3}\right), 1.6$ - $1.3\left(2 \mathrm{H}, \mathrm{CH}_{2}\right), 2.7$ - $2.4(1 \mathrm{H}, \mathrm{CH}-$

ICPP), $1.5-1.2\left(6 \mathrm{H}, \mathrm{CH}\left(\mathrm{CH}_{3}\right)_{2}\right) ;{ }^{13} \mathrm{C}-\mathrm{NMR} \delta$ 174-166 $(\mathrm{C}=\mathrm{O}), 152-100(\mathrm{Ph}), 117-115$

$(\mathrm{CN}), 68$ - $58(\mathrm{OCH}), 60$ - $54\left(\mathrm{OCH}_{3}\right), 54-41\left(\mathrm{CH}, \mathrm{CH}_{2}\right), 25$ - $23\left(\mathrm{CH}\left(\mathrm{CH}_{3}\right)_{2}\right) ;$ FTIR

$\left(\mathrm{cm}^{-1}\right)$ : 3077-2869 (m, C-H), 2222 (m, CN), 1740 (s, C=O), 1238 (s, C-O-C), 978 (s, C-H out of plane). Nitrogen content $2.64 \%$. 
Table 1. Molecular characteristics of ST-IPCA copolymers

\begin{tabular}{|c|c|c|c|c|}
\hline $\mathrm{R}$ & $\begin{array}{c}\text { Conversion } \\
\%\end{array}$ & $\begin{array}{c}\text { Nitrogen } \\
\text { wt\% }\end{array}$ & $\begin{array}{c}\text { \% mole } \\
\text { ST }\end{array}$ & $\begin{array}{c}\text { \% mole } \\
\text { IPCA }\end{array}$ \\
\hline $2,3-\left(\mathrm{CH}_{3}\right)_{2}$ & 12.1 & 0.15 & 79.5 & 20.5 \\
\hline $2,4-\left(\mathrm{CH}_{3}\right)_{2}$ & 14.2 & 0.14 & 81.9 & 18.1 \\
\hline $3,5-\left(\mathrm{CH}_{3}\right)_{2}$ & 12.8 & 0.19 & 72.6 & 27.4 \\
\hline $2,3-\left(\mathrm{CH}_{3} \mathrm{O}\right)_{2}$ & 12.7 & 0.16 & 75.5 & 24.5 \\
\hline $2,4-\left(\mathrm{CH}_{3} \mathrm{O}\right)_{2}$ & 11.2 & 0.13 & 81.5 & 18.5 \\
\hline $2,5-\left(\mathrm{CH}_{3} \mathrm{O}\right)_{2}$ & 13.4 & 0.17 & 74.4 & 25.6 \\
\hline $2,6-\left(\mathrm{CH}_{3} \mathrm{O}\right)_{2}$ & 15.1 & 0.13 & 81.5 & 18.5 \\
\hline $3,4-\left(\mathrm{CH}_{3} \mathrm{O}\right)_{2}$ & 14.1 & 0.15 & 77.7 & 22.3 \\
\hline $3,5-\left(\mathrm{CH}_{3} \mathrm{O}\right)_{2}$ & 12.7 & 0.19 & 69.9 & 30.1 \\
\hline
\end{tabular}

The copolymers prepared in the present work are all soluble in ethyl acetate, THF, DMF and $\mathrm{CHCl}_{3}$ and insoluble in methanol, ethyl ether, and petroleum ether.

\section{Conclusions}

Novel dimethyl and dimethoxy ring-disubstituted isobutyl phenylcyanoacrylates were prepared and copolymerized with styrene. The compositions of the copolymers were calculated from nitrogen analysis and the structures were analyzed by $\mathrm{IR}, \mathrm{H}^{1}$ and ${ }^{13} \mathrm{C}$ NMR. 


\section{Acknowledgments}

The authors are grateful to acknowledge that the project was partly supported by Chicago Society of Coating Technology (CSCT).

\section{References}

1. Novel Application of Polymer Networks Carrying Tertiary Amines as a Catalyst Inside Microflow Reactors Used for Knoevenagel Reactions. Berg, Patrik; Obst, Franziska; Simon, David; Richter, Andreas; Appelhans, Dietmar; Kuckling, Dirk. European Journal of Organic Chemistry (2020), 2020(35), 5765-5774.

2. Efficient and reusable amine-functionalized polyacrylonitrile fiber catalysts for Knoevenagel condensation in water. Li, Guowei; Xiao, Jia; Zhang, Wenqin. Green Chemistry (2012), 14(8), 2234-2242.

3. A novel and green method for the synthesis of highly substituted isoquinoline derivatives in ionic liquid. Wang, Xiang-Shan; Wu, Jian-Rong; Li, Qing; Zhang, Mei-Mei. Journal of Heterocyclic Chemistry (2009), 46(6), 1355-1363.

4. Catalyst-Free [3+3] Annulation/Oxidation of Cyclic Amidines with Activated Olefins:

When the Substrate Olefin Is Also an Oxidant. Han, Wendan; Li, Yuanhang;

Raveendra Babu, Kaki; Li, Jing; Tang, Yuhai; Wu, Yong; Xu, Silong. Journal of Organic Chemistry (2021), 86(11), 7832-7841.

5. N,N'-Dioxide-Lanthanum(III)-Catalyzed Asymmetric Cyclopropanation of 2-Cyano-3arylacrylates with 2-Bromomalonates. Zhang, Yuheng; Lin, Lili; Chen, Yushuang; Liu, 
Xiaohua; Feng, Xiaoming. Advanced Synthesis \& Catalysis (2017), 359(11), 18311836.

6. Highly efficient synthesis of pyranoquinoline derivatives catalyzed by piperidine. Kaur, Jasreen; Bariwal, Jatinder; Bedi, Preet Mohinder Singh; Kaur, Maninderjit; Kapoor, Amit; Kaur, Mandeep; Verma, Poonam. World Journal of Pharmacy and Pharmaceutical Sciences (2017), 6(12), 850-870.

7. Stereoselective synthesis of polysubstituted cyclopropanes from poly(ethylene glycol) supported pyridinium ylide. Zhao, Pan; Lu, Cui-fen; Yang, Gui-chun; Chen, Zu-xing; Dong, Nian-guo; Shi, Jia-wei. Chemical Research in Chinese Universities (2011), 27(6), 984-987.

8. Some derivatives of o-vanillin. Perkin, William H., Jr.; Robinson, Robert. Journal of the Chemical Society, Transactions (1914), 105, 2376-92.

9. Microwave enhanced Knoevenagel condensation of ethyl cyanoacetate with aldehydes. Chung, Sung-Kee; Chang, Young-Tae. Synthetic Communications (1997), 27(4), 533541.

10. One-pot esterification and amide formation via acid-catalyzed dehydration and Ritter reactions. Dawar, Pankaj; Raju, M. Bagavan; Ramakrishna, Ramesha Andagar. Synthetic Communications (2014), 44(6), 836-846.

11. Synthesis of pyrido[2,1-c]-1,2,4-triazine, 1,2,4-triazolo[4,3-a]pyridine and 2(substituted-pyrazolyl)nicotinonitrile and their effect on Biomphalaria alexandrina snail enzymes. Swelam, S. A.; Fathalla, O. A.; Zak, M. E. A.; Aly, H. F. Egyptian Journal of Chemistry (2004), 47(6), 677-692. 
12. Synthesis of dihydrouracils spiro-fused to pyrrolidines: drug-like molecules based on the 2-arylethyl amine scaffold. Blanco-Ania, Daniel; Valderas-Cortina, Carolina;

Hermkens, Pedro H. H.; Sliedregt, Leo A. J. M.; Scheeren, Hans W.; Rutjes, Floris P. J. T. Molecules (2010), 15, 2269-2301.

[13] Novel Copolymers of Styrene. 14. Some Ring-Disubstituted Methyl 2-Cyano-3Phenyl-2-Propenoates. G.B. Kharas, A. Shahbein, J.L. Fabbri, C.E. Fanter, D.R. Garcia, M.G. Ibarra, D. Lane, E. Marshall, E.E. Pierce, I.F. Sanchez, B.C. Sandoval, and A.D. Santi. J. Macromol. Sci. A51(5) 394-398 (2014).

[14] Novel Copolymers of Styrene and Dialkoxy Ring-substituted Methyl 2-cyano-3phenyl-2-propenoates. G.B. Kharas, J.L. Christensen, D.J. Cichanski, K.E. Goldman, C.L. Gordon, L.M. Knowles, C.N. Krefft, M. Matouk, and K. Watson. J. Macromol. Sci., 43 (7) 989-994 (2006).

[15] Novel Copolymers of Styrene. 4. Alkyl Ring-substituted Ethyl 2-Cyano-3-phenyl-2propenoates. G.B. Kharas, E.S. Molina, E.E. Pierce, S.A.B. Cocjin, C. Cruz, K.M. Fair, S.S. Flaksman, M.J. Liggins, A.D. Meglei, M.E. Pantos, and G.C. Pisano. J.Macromol. Sci. A50 (2) 144-148 (2013).

[16] Novel Copolymers of Styrene. 6. Alkoxy Ring-substituted Ethyl 2-Cyano-3-phenyl-2propenoates.G.B. Kharas, A.A. Delgado, N.E. Anderson, A. Bajor, A.C. Colbert, A. Coleman, C.E. Gregory, J. Hayes, J. Lantin, J.M. Malecki, T.C. Murphy, A. Oprescu, B.F. Rydzon, and I. Timoshevskaya. J.Macromol. Sci. A50 (3) 276-280 (2013).

[17] Novel Copolymers of Styrene. 2. Some Ring-substituted Ethyl 2-Cyano-3-phenyl-2propenoates. G. B. Kharas, E.S. Molina, B. Bobot, J. Bueno, J. Carney, J.Y.C. Chung, 
K.N. Krerowicz, T. Miller, S.L.I. Mills, M.A. Stankovich, I. Syed, C.S. Vaidya, and P. Wesolowski. J. Macromol. Sci. A50 (1) 1-5 (2013).

[18] Novel Copolymers of Styrene. 5. Alkyl and Alkoxy Ring-Disubstituted Propyl 2Cyano-3-Phenyl-2-Propenoates. G.B. Kharas, W.M.B. Barros, A. Affaneh, L.A. Alyahya, A.S. Ansari, M.J. Asztalos, P.T. Burns, Y. Cardoza, K.J.O. Galvan, K.J. Hall, S.M. Short, and M.A. Sovereign. J. Macromol. Sci. A53(11) 664-668 (2016).

[19] Novel Copolymers of Styrene. 6. Some Ring-Disubstituted Propyl 2-Cyano-3-Phenyl2-Propenoates. G.B. Kharas, L.A. Alyahya, S. M. Rocus, A. Ismail, R. Juarez, A. Kavaliauskaite, K.Y. Lechuga, A.C. Leeper, and G.C. Lenti. J. Macromol. Sci. A53(12) 725-728 (2016).

[20] Synthesis and styrene copolymerization of dimethyl and dimethoxy ring-substituted isopropyl cyanophenyl propenoates. R.L. Pride, A. MacDonald, E.E. McCarthy, A.N. Modi, E. Mucciolo, A.L. Schinderle, S. Trewartha, J.D. Tylka, M. Wade, J. Wat, W.S. Schjerven and G.B. Kharas. Academic J Polym Sci. 3(1): 555602 (2019).

DOI:10.19080/AJOP.2019.02.555602

[21] Synthesis and styrene copolymerization of novel oxy ring-disubstituted isopropyl phenylcyanoacrylates. J.L. Zepeda, M.M. Betts, S. Dalloul, A.G. Gonzalez, J.K. Henning, R.X. Liu, J.J. Ludtke, J.R. Mihai, S.M. Rocus, W.S. Schjerven, and G.B. Kharas. J. Phys. Chem. Res. 1(2) 1-5 (2019).

[22] Synthesis and styrene copolymerization of novel ring-disubstituted isopropyl cyanoacrylates. P.M. Whelpley, S. Bajramovic, D.M. Bracamontes, G.A. Buechner, 
A.D. Eremin, E.J. Kowalczyk, D.D. Lender, R. McCann, W.S. Schjerven, G.B.

Kharas. ChemRxiv (2019), 1-15. https://doi.org/10.26434/chemrxiv.9891161.v1.

[23] Novel Copolymers of Styrene. 6. Some Ring-Disubstituted Butyl 2-Cyano-3-Phenyl-2-

Propenoates. G.B. Kharas, S.M. Rocus, M. Zuziak, V. Elangovan, A.N. Kovaleva, S. Malik, O. Nwosu, L.A. Patel, A. Piche, and S.J. Rosengarden. J. Macromol. Sci. A52 (12) 971-975 (2015).

[24] Novel Copolymers of Styrene. 5. Methyl and Methoxy Ring-Disubstituted Butyl 2Cyano-3-Phenyl-2-Propenoates. G.B. Kharas, H. Feng, L.A. Arendt, S.E. Belton, M.Q. Edwards, E.K. Franz, A. Grin, C.M. Hale, I.C. Oyeyipo, A.L. Wolske, P.L. Zavala, and A. Zenunovic. J. Macromol. Sci. A52 (12) 976-981 (2015).

[25] Synthesis and styrene copolymerization of novel trisubstituted ethylenes: 6. Methyl and methoxy ring-disubstituted 2-methoxyethyl 2-cyano-3-phenyl-2-propenoates. Sean F. Bobrov, Elizabeth J. Bruce, Kailee J. Buttice, Eduardo Cortes, Feona M. Cotter, Kathleen M. Fortune, Svetlana Galkina, Nick Goedert, Erin D. Jurgerson, Kelly J. Mcgowen, Maximilian J. Nufer, Esha K. Patel, Sara M. Rocus, William S. Schjerven, and Gregory B. Kharas. ChemRxiv. Preprint. (19.01.2021). https://doi.org/10.26434/chemrxiv.13262660.v6

[26] Synthesis and styrene copolymerization of novel trisubstituted ethylenes: 5. Dimethyl and dimethoxy ring-substituted octyl phenylcyanoacrylates. Martin S. Wasilewski, Grant W. Boyson, Georgina N. Canavesio, Sarah N. Keaton, Matthew R. Lee, Luke Meyer, Zoe A. Ryan, Eric Seeger, Mahmood I. Shah, Rebeca M. Tojo Suárez, Anahi F. Toolabian, Bernadette C. Tudor, Sara M. Rocus, William S. Schjerven, and Gregory 
B. Kharas. ChemRxiv. Cambridge Open Engage Version 16 Jul 07, 2021.

https://doi.org/10.33774/chemrxiv-2021-k812v-v16

[27] SciFinder Structure Search Feb 15, 2022.

[28] Synthesis and styrene copolymerization of novel alkyl ring-substituted isobutyl 2cyano-3-phenyl-2-propenoates. Schjerven, William S.; Groy, Randi; Antonishina, Yekaterina S.; Black, Claire I.; Bolin, Madysen P.; Caniglia, John; Daroach, Deepak P.; Farooqui, Farah T.; Feiz, Yalda; Garcia, Sam; Gasparini, Gabriella; Kharas, Gregory B. Abstracts of Papers, 259th ACS National Meeting \& Exposition, Philadelphia, PA, United States, March 22-26, (2020), POLY-0388.

[29] Smith, M. B.; March, J. Addition to Carbon-Hetero Multiple Bonds, In March's Advanced Organic Chemistry, J. Wiley \& Sons: New York, Ch.16, 1225 (2001). 